\title{
Vibration Analysis of Rotating Wind Turbine Blades Based on Piezoelectric Materials
}

\author{
Ben Jing and Wang Hao \\ Shanghai University of Electric Power, Shanghai, China.
}

(Received 16 June 2020; accepted 18 December 2020)

Piezoelectric materials have a piezoelectric effect that converts mechanical energy into electrical energy. In this paper, the blades of the rotating wind turbine are simplified as flexible beams fixed on the rotating wheels, and piezoelectric ceramics are added to the beams as sensors and actuators respectively to establish an analysis model of the vibration behavior of the piezoelectric sandwich rotating wind turbine blades. Based on Newton's second law, different accelerations are added to the rotating wheel to obtain the differential equation of a vibration variable coefficient. The fourth order Runge-Kutta method is used to solve variable coefficient differential equations. The hypothetical modal method is applied to solve the displacement of the free end of the flexible beam. A numerical simulation is also carried out to analyse the magnitude and change trend of the voltage output by adding piezoelectric materials at different angular velocities. The results show that the greater the rotational angular velocity, the greater the displacement of the free end of the flexible beam, and the greater the voltage due to the piezoelectric effect of the piezoelectric material. When the rotation angular velocity reaches a stable value, the displacement of the free end and the generated voltage will also reach a stable value.

\section{INTRODUCTION}

Piezoelectric materials are an important class of functional materials that have a piezoelectric effect that converts mechanical and electrical energy into each other. Wind turbine blades are not rigid. Under the action of randomly flowing aerodynamics, the blades will also vibrate and elastically deform while rotating. Therefore, as in previous studies, it cannot be directly reduced to a static beam or plate to analyse its vibration. In this paper, the rotating wind turbine blade was simplified as a cantilever beam of an equal cross section connected to the rotating wheel, and piezoelectric ceramics were added above and below the beam. According to Newton's second law, the vibration flexible coefficient differential equation is obtained by analyzing the force of the rotating flexible beam. The fourth order Runge-Kutta method was used to solve the vibration variable coefficient differential equation. The hypothetical modal method was used to solve the free end displacement of the beam due to aeroelasticity, and the characteristics of the free end displacement are simulated and observed. Apply different angular velocities to the rotating wheel and observe whether the piezoelectric cantilever beam can convert mechanical energy into electrical energy under the piezoelectric material sensing effect, whether there is a voltage output, and how the output voltage has varying characteristics. ${ }^{1-3}$ The results show that the dynamic strain induced by the piezoelectric ceramic layer in such a piezoelectric energy harvester will produce an $\mathrm{AC}$ voltage output on the piezoelectric ceramic layer. ${ }^{4}$ The greater the rotation angular velocity, the greater the displacement of the free end and the greater the output voltage. When the rotation angular velocity reaches a stable value, the free-end displacement and output voltage also tend to stabilize.

To clearly represent the electrical output, Luet et al. ${ }^{5}$ used the vibration mode shape obtained from the Euler-Bernoulli beam theory and the piezoelectric constitutive relationship. The output was linked to the mechanical mode shape. Ajitsariaet et al. ${ }^{6}$ proposed a bimorph piezoelectric cantilever model. They tried to combine the static sensing / driving equation (with constant radius of curvature and static tip force) with the dynamic Euler-Bernoulli beam equation (where the curvature The radius is variable. Under basic excitation, there is no tip force). ${ }^{7}$

\section{ROTATION ANALYSIS OF FLEXIBLE BEAM BASED ON PIEZOELECTRIC MATERIAL}

Wind turbine blades are flexible components that move in a large range. Under the conditions of relatively large wind speeds, the rapidly rotating blades will affect the blade's lateral movement under the action of the blade's axial tension or axial pressure, resulting in bending resistance. As the rigidity is strengthened and weakened, and the frequency of lateral elastic vibration becomes larger/smaller. This is the so-called "dynamic rigidity" and "dynamic weakening" phenomena. ${ }^{8}$ For the wind turbine blades with piezoelectric materials added, the sensing and actuating characteristics of the piezoelectric materials themselves will effectively reduce the influence of the axial force on the bending rigidity and lateral vibration of the blades. In this paper, the displacement of the cantilever beam at the free end caused the piezoelectric material to produce a sensing effect and the output voltage was obtained. 


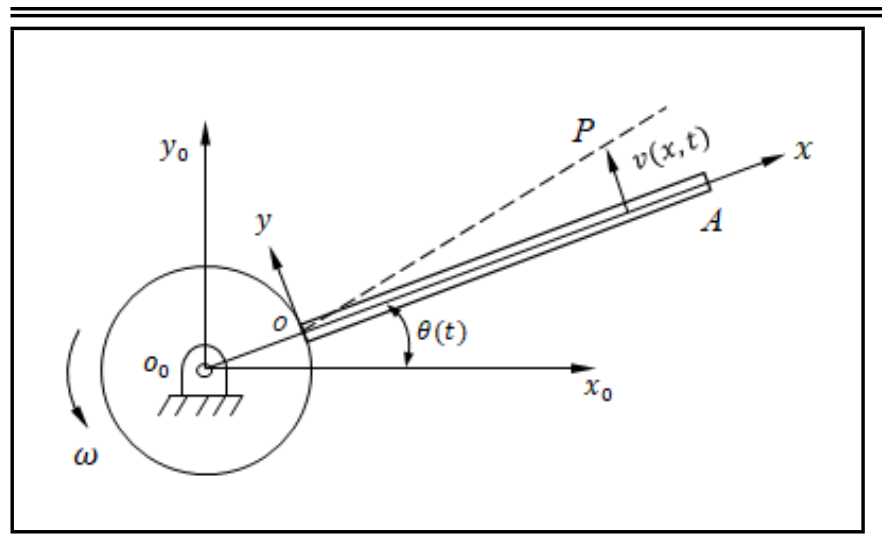

Figure 1. Model of flexible beam connecting rotating wheel.

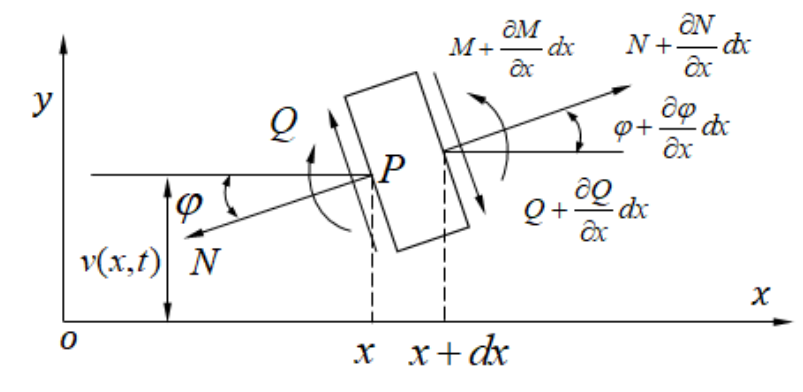

Figure 2. Force diagram of beam micro-segment considering axial force.

\subsection{Mathematical Modeling of Cantilever Beam-Establish Differential Equation of Vibration}

The structure of the wind turbine blades and tower was simplified as a flexible beam of an equal cross-section connected to a rotating wheel with known rotation laws. ${ }^{4,9,10}$ Under normal operating conditions, the model established by connecting the flexible cantilever beam to the rotating wheel is shown in Fig. 1.

Perform a force analysis on the flexible beam under the model and take a small section of the beam. The force analysis diagram is shown in Fig. 2.

In Fig. 2, $N, Q$ and $M$ were expressed as axial force, shear force and bending moment, respectively.

According to Newton's second law, the force analysis was performed along the $y$ direction, and the differential equation of motion as shown in Eq. (1) was obtained.

$$
\begin{aligned}
&(\rho A d x) a_{p y}=-N \sin \varphi+Q \cos \varphi \\
&+\left(N+\frac{\partial N}{\partial x}\right) \sin \left(\varphi+\frac{\partial \varphi}{\partial x} d x\right) \\
& \quad-\left(Q+\frac{\partial Q}{\partial x} d x\right) \cos \left(\varphi+\frac{\partial \varphi}{\partial x} d x\right)
\end{aligned}
$$

where, $\rho$ was the density of the beam, $A$ was the crosssectional area of the flexible beam, $a_{p y}$ was the projection of the absolute acceleration of the centroid $P$ of the left side of the selected beam micro-segment along the $y$-axis direction, $\varphi$ was the elastic corner of the left end of the beam micro-end caused by bending deformation.

Since the displacement of $P$ along the $x$-axis direction can be ignored in bending deformation, the expression was obtained as shown in Eq. (2).

$$
a_{p y}=\frac{\partial^{2} v}{\partial t^{2}}+\ddot{\theta}(r+x)
$$

where, $\ddot{\theta}(r+x)$ was expressed as tangential acceleration, $\theta$ was the rotation angle of the rotating wheel, $r$ was the wheel radius, $v(x, t)$ was the lateral displacement of point $P$. The relationship between shear force $Q$ and lateral displacement $v(x, t)$ is shown in Eq. (3).

$$
Q=E I \frac{\partial^{3} v}{\partial x^{3}}
$$

where, $E$ was the elastic modulus and $I$ was the moment of inertia of the cross section of the beam. In the case of a small deformation of the beam, the elastic angle $\varphi$ of the elastic surface of the beam was a small amount, so the relationship shown in Eq. (4) is stored.

$$
\varphi \approx \frac{\partial v}{\partial x}
$$

Ignoring the second-order trace term of $d x$, Eq. (1) can be simplified to obtain Eq. (5).

$$
\rho A\left[\frac{\partial^{2} v}{\partial t^{2}}+\ddot{\theta}(r+x)\right]=-E I \frac{\partial^{4} v}{\partial x^{4}}+\frac{\partial(N \varphi)}{\partial x} .
$$

In the case of small deformation of the beam, there is the relationship of Eqs. (6) and (7).

$$
N \approx \bar{N}
$$

$$
\bar{N}=\rho A(l-x) \dot{\theta}^{2}\left(r+\frac{l+x}{2}\right) .
$$

Combining the above equations, we can get Eq. (8).

$$
\begin{array}{r}
E I \frac{\partial^{4} v}{\partial x^{4}}+\rho A\left\{\frac{\partial^{2} v}{\partial t^{2}}\right. \\
+\dot{\theta}^{2}\left[(r+x) \frac{\partial v}{\partial x}-\frac{1}{2}(l-x)(2 r+l+x) \frac{\partial^{2} v}{\partial x^{2}}\right] \\
+\ddot{\theta}(r+x)\}=0 .
\end{array}
$$

Since this was a linear partial differential equation with variable coefficients, the approximate solution of the displacement $v(x, t)$ at the free end of the beam was solved using the assumed modal method. ${ }^{11}$

The displacement of the free end of the beam can be expressed as Eq. (9). 


$$
v(x, t)=\sum_{i=1}^{n} Y_{i}(x) q_{i}(t) .
$$

Equation (9) where $Y_{i}(x)$ was the assumed modal function, $q_{i}(t)$ was the corresponding generalized coordinate, and $n$ was the number of selected assumed modal functions. Here, the modal function of the cantilever beam with an equal crosssection was selected as the assumed modal function $Y_{i}(x)$, and its expressions were Eqs. (10)-(12).

$$
\begin{aligned}
& Y_{i}(x)= \\
& \cos \left(\beta_{i} x\right)-\cosh \left(\beta_{i} x\right)+\gamma_{i}\left(\sin \left(\beta_{i} x\right)-\sinh \left(\beta_{i} x\right)\right) ; \\
& \beta_{1} l=1.875, \beta_{2} l=4.694, \beta_{i} l \approx(i-0.5) \pi \\
& \quad(i=3, \ldots, n) ; \\
& \gamma_{i}=-\frac{\cos \left(\beta_{i} l\right)+\cosh \left(\beta_{i} l\right)}{\sin \left(\beta_{i} l\right)+\sinh \left(\beta_{i} l\right)}, \quad(i=1,2 \ldots, n) .
\end{aligned}
$$

Substitute the free end displacement expression of the beam into the beam's lateral elastic vibration differential equation, multiply both sides of the equation by $Y_{j}(x)(j=1,2, \ldots, n)$, and then take a definite integral along the length of the beam (the orthogonality of the modal function was considered during integration) to obtain the expression for Eq. (13).

$$
\begin{array}{r}
m_{j} \ddot{q}_{j}(t)+\left(k_{j}-\dot{\theta}^{2} m_{j}\right) q_{j}(t)-\dot{\theta}^{2} \sum_{i=1}^{n} b_{j i}+c_{j} \ddot{\theta}=0 \\
(j=1,2, \ldots, n) .
\end{array}
$$

The differential equation was a variable coefficient differential equation, and the coefficient expressions in the equation are Eqs. (14)-(17).

$$
\begin{gathered}
m_{j}=\rho A \int_{0}^{l}\left[Y_{j}(x)\right]^{2} d x \\
k_{j}=\int_{0}^{l} E I Y_{j}(x) Y_{j}^{4}(x) d x \\
b_{j i}=\rho A \int_{0}^{l}\left[(l-x)(2 r+l+x) Y_{i}^{\prime \prime}(x)\right] d x \\
c_{j}=\rho A \int_{0}^{l}(r+x) Y_{j}(x) d x .
\end{gathered}
$$

Both the generalized coordinate $q_{j}(0)$ and the generalized velocity $\dot{q}_{j}(0)$ at the initial moment were determined according to the initial state of the flexible beam $v(x, 0)$ and $\dot{v}(x, 0)$, the expressions are Eqs. (18) and (19).

$$
q_{j}(0)=\frac{\rho A}{m} \int_{0}^{l} Y_{j}(x) v(x, 0) d x ;
$$

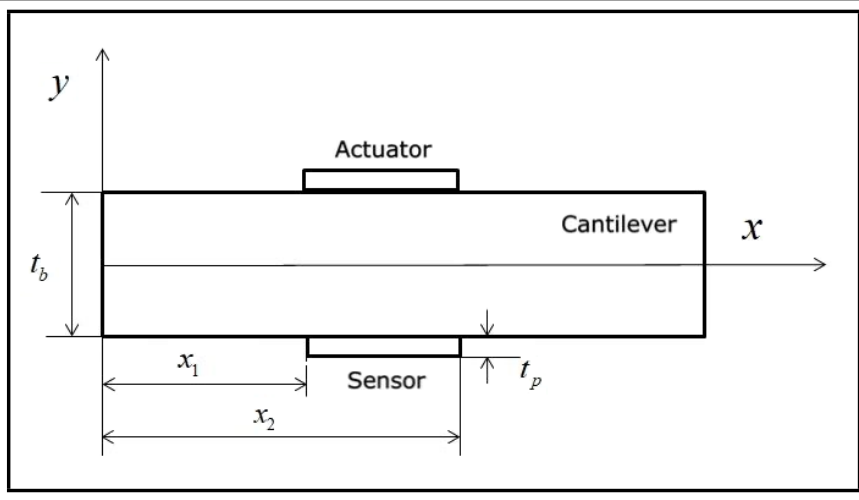

Figure 3. Cantilever beam model with piezoelectric plate added.

$$
\dot{q}_{j}(0)=\frac{\rho A}{m} \int_{0}^{l} Y_{j}(x) \dot{v}(x, 0) d x .
$$

Within a certain timeframe, the angular velocity of the rotating wheel gradually increased with time. When the rotational wheel reached stability, the angular velocity of the rotating wheel was a certain value that would not change with time.

\subsection{Cantilever Beam Model With Piezoelectric Material Added-Dynamic Analysis of Sensing Characteristics of Piezoelectric Cantilever Beam}

We pasted a pair of piezoelectric plates on the upper and lower surfaces of the cantilever beam as sensors and actuators, respectively. The length and thickness of the piezoelectric plates were $L_{p}$ and $t_{p}$, and $x_{1}$ and $x_{2}$ were the distance from the piezoelectric plate to the rotating wheel. As shown in Fig. 3.

According to the analysis of the approximate differential equation of the deflection axis under the small deformation of the symmetric bending of the beam in material mechanics, we can obtain Eqs. (20) and (21).

$$
\begin{aligned}
& T_{1}(x, t)=\frac{p E_{p}}{\rho} ; \\
& \frac{1}{\rho}=\frac{\partial^{2} \nu(x, t)}{\partial^{2} x}
\end{aligned}
$$

where, $T_{1}$ was the stress on the beam along the $x$-axis, unit: $\mathrm{N} / \mathrm{m}^{2}, \rho$ was the radius of curvature of the bending deformation, unit: $\mathrm{m}, E_{p}$ was the elastic modulus of the piezoelectric sheet, unit: $\mathrm{Pa}$.

In this model, ignoring the influence of the adhesive layer of the piezoelectric sheet on the vibration characteristics of the cantilever beam, the stress of the piezoelectric sheet was the stress of the cantilever beam. Therefore, the expression of the positive piezoelectric effect is Eq. (22).

$$
D=d T
$$

where, $D$ was electrical displacement, $d$ was piezoelectric constant strain matrix, $T$ was stress. 
Therefore, the electrical displacement under the positive piezoelectric effect is shown in Eq. (23).

$$
D_{3}=\varepsilon_{33} E_{3}+d_{31} T_{1} .
$$

In piezoelectric materials as sensors, the electric field strength $E_{3}=0$, so in the $z$-axis direction, the electrical displacement is as shown in Eq. (24).

$$
D_{3}(x, t)=d_{31} T_{1}(x, t)=p d_{31} E_{p} \frac{\partial^{2} \nu(x, t)}{\partial^{2} x} ;
$$

According to the theory of natural mode expansion, the motion deflection $p$ of the beam can be expressed by the linear combination of its natural mode $\left\{Y_{i}\right\}$ as shown in Eq. (25).

$$
p=p\left(L_{b}, t\right)=\sum_{i=1}^{n} Y_{i}\left(L_{b}\right) q_{i}(t)=Y_{L} q ;
$$

Therefore, the electrical displacement is expressed as Eq. (26).

$$
D_{3}=\frac{h}{2} E_{p} d_{31} \sum_{i=1}^{n} Y_{i}^{\prime \prime}\left(L_{b}\right) q_{i}(t) .
$$

Since the width of the piezoelectric plate was the same as the width of the cantilever beam, the coordinates of the left and right ends of the piezoelectric plate on the $x$-axis were $x_{1}$ and $x_{2}$. The amount of charge on the piezoelectric material surface can be expressed as Eqs. (27) and (28).

$$
\begin{gathered}
Q(x, t)=\int_{x_{1}}^{x_{2}} D_{3}(x, t) b d x= \\
\int_{x_{1}}^{x_{2}} \frac{h E_{p} d_{31}}{2} b \sum_{i=1}^{n} Y_{i}^{\prime \prime}(x) q_{i}(t) d x \\
Q(x, t)=\frac{h E_{p} d_{31}}{2} b\left[Y^{\prime}\left(x_{2}\right)-Y^{\prime}\left(x_{1}\right)\right] q_{i}(t)
\end{gathered}
$$

Therefore, the voltage generated on the two surfaces of the piezoelectric sensor can be expressed as Eq. (29).

$$
\begin{aligned}
& U_{S}(x, t)=\frac{Q(x, t)}{C_{P}}= \\
& \quad \frac{h E_{p} d_{31}}{2 C_{P}} b\left[Y^{\prime}\left(x_{2}\right)-Y^{\prime}\left(x_{1}\right)\right] q_{i}(t) ;
\end{aligned}
$$

where, $C_{p}$ was the capacitance of the piezoelectric sheet, which was a known quantity and its unit was farad.

\section{DISPLACEMENT RESPONSE OF FREE END OF FLEXIBLE BEAM}

Select a set of parameters and use the rotating flexible beam model with the piezoelectric material added above for simulation research. The specific parameters of the flexible cantilever are shown in Table 1.
Table 1. Material parameters of flexible beam.

\begin{tabular}{||l|l|}
\hline Parameter & Numerical value \\
\hline Rotating wheel radius $(\mathrm{m})$ & 0.55 \\
\hline Flexible beam length $(\mathrm{m})$ & 0.9 \\
\hline Beam cross-sectional area $\left(\mathrm{m}^{2}\right)$ & $3.18 \cdot 10^{-5}$ \\
\hline Moment of inertia $\left(\mathrm{m}^{4}\right)$ & $2.65 \cdot 10^{-12}$ \\
\hline Density $\left(\mathrm{kg} \cdot \mathrm{m}^{-3}\right)$ & 7866 \\
\hline Elastic Modulus $\left(\mathrm{N} \cdot \mathrm{m}^{-2}\right)$ & $2.01 \cdot 10^{11}$ \\
\hline
\end{tabular}

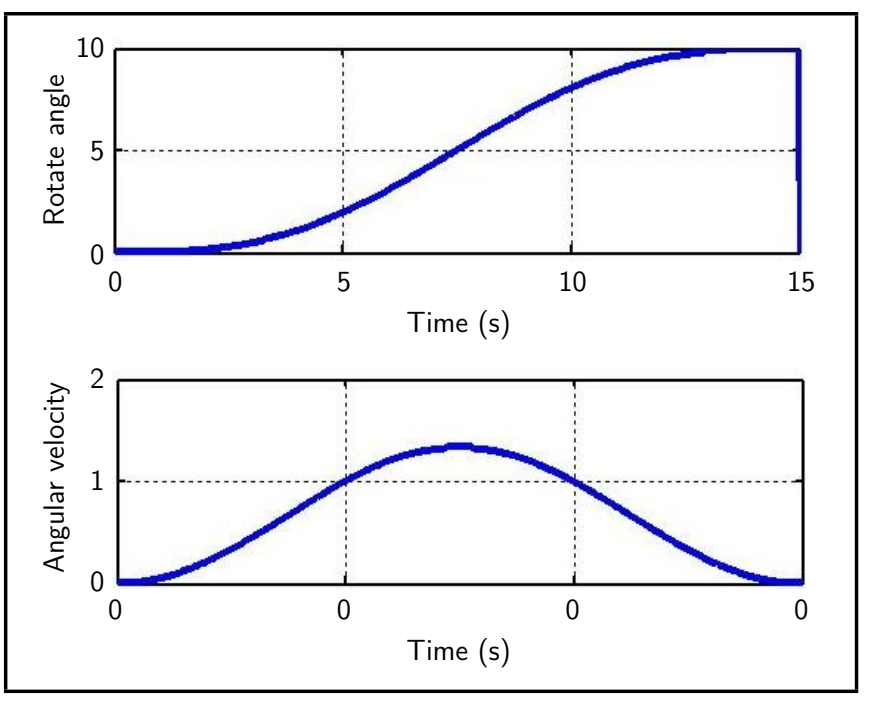

Figure 4. Rotation wheel rotation angle and angular acceleration with time.

For the piezoelectric sheet, its parameters were: elastic modulus $E_{p}=6.3 \times 10^{10} \mathrm{~Pa}$, piezoelectric constant $d_{31}=$ $-1.2 \times 10^{-10} \mathrm{C} / \mathrm{N}$, piezo thickness $t_{p}=0.0003 \mathrm{~m}$, width of flexible beam $b=0.1 \mathrm{~m}$.

The initial lateral displacement and lateral velocity of the flexible beam were both 0 , and the angular velocity equation of the rotating wheel is Eq. (30).

$$
\dot{\theta}=\left\{\begin{array}{lr}
\frac{\omega_{0}}{T} t-\frac{\omega_{0}}{2 \pi} \sin \left(\frac{2 \pi}{T} t\right) & 0 \leq t \leq T \\
\omega_{0} & t \geq T
\end{array} .\right.
$$

In the Eq. (30), the number of modal functions was taken to be 2 during the solution process using the hypothetical modal method. In addition, it can be seen from Fig. 4 that when $T=$ $7.5 \mathrm{~s}$, the angular velocity of the rotating wheel reached the peak value; $T=15 \mathrm{~s}$, when $t=T$ in the formula, the angular velocity of the rotating wheel reached the steady state angular velocity.

The rotation angular speed of the rotating wheel was $10 \mathrm{rad} / \mathrm{s}$ and $15 \mathrm{rad} / \mathrm{s}$.

When the rotational angular velocity was $10 \mathrm{rad} / \mathrm{s}$, the curve of the generalized displacement and velocity of the free end of the beam with time at $j=1$ is shown in Fig. 5 .

The curve of the generalized displacement and velocity of the free end of the beam with time at $j=2$ is shown in Fig. 6 .

The curve of the vibration displacement of the free end of the beam with time is shown in Fig. 7. 


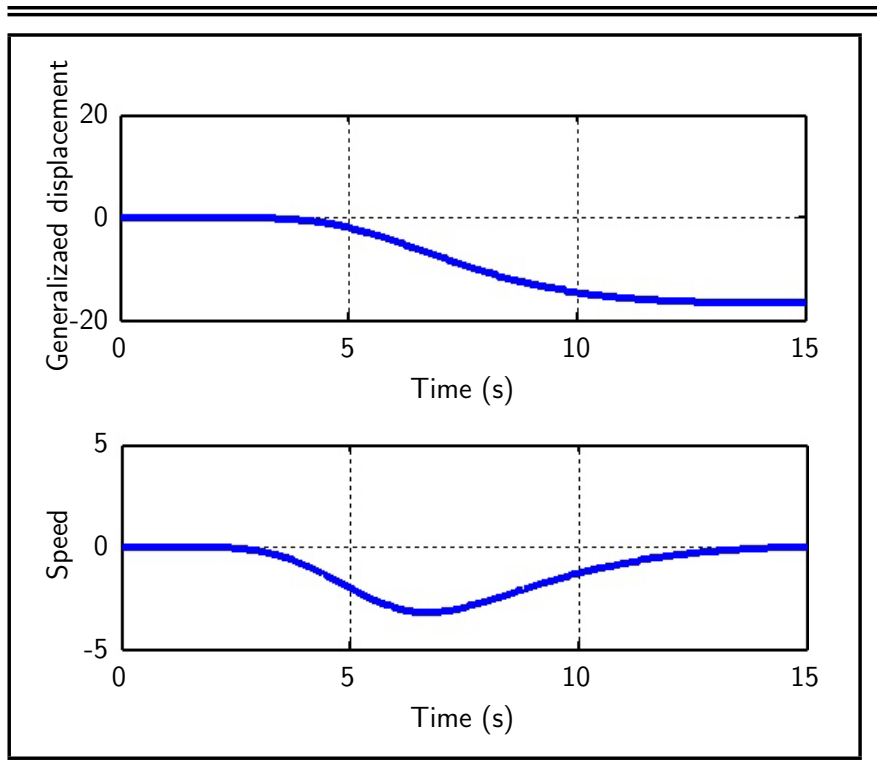

Figure 5. When $j=1$, the displacement and velocity of the free end of the flexible beam.

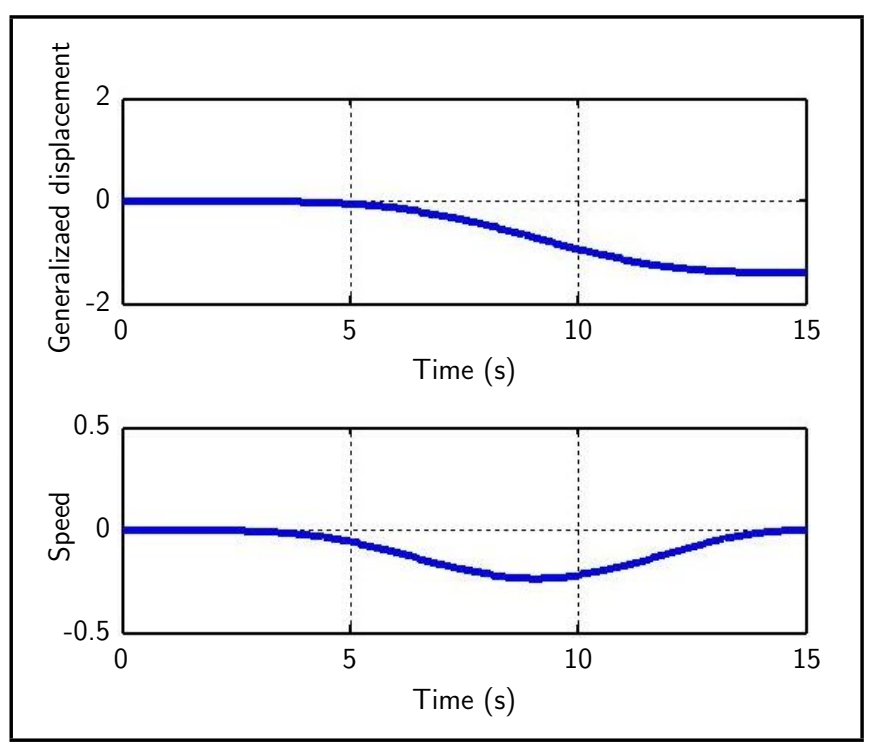

Figure 6. When $j=2$, the generalized displacement and velocity of the free end of the flexible beam.

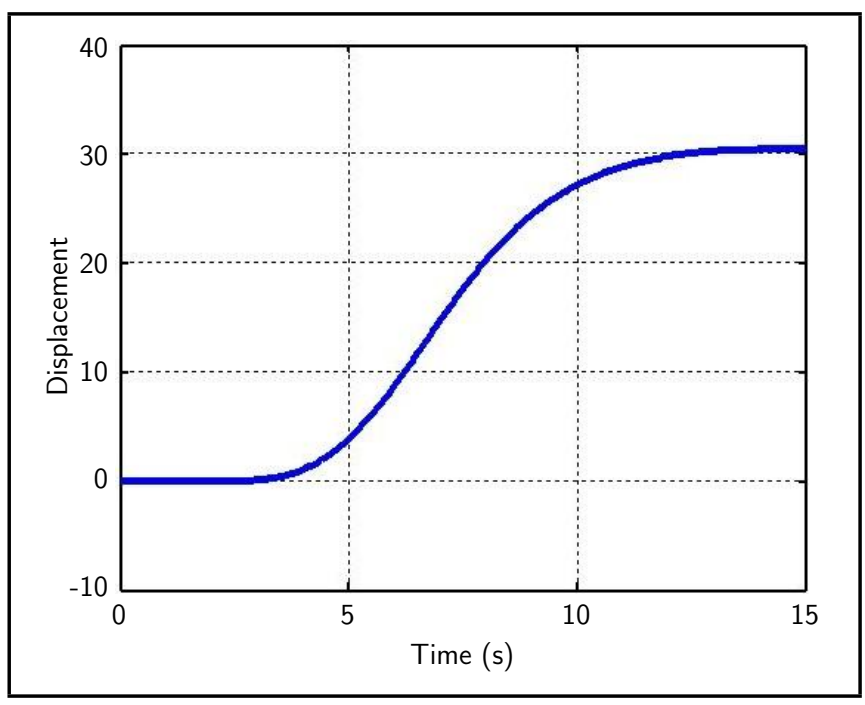

Figure 7. Vibration displacement of free end of flexible beam.
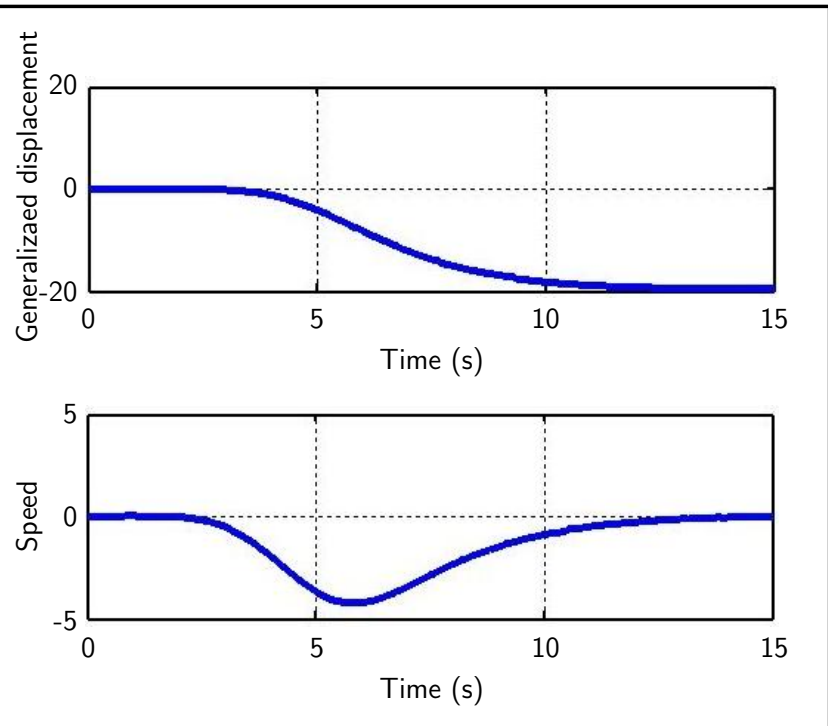

Figure 8. When $j=1$, the generalized displacement and velocity of the free end of the flexible beam.

When the rotating angular velocity of the rotating wheel was $10 \mathrm{rad} / \mathrm{s}$, the fourth order Runge Kuta was used to solve the differential equation of the vibration variable coefficient of the established flexible beam. It can be seen from Figs. 5 and 6 that when $j=1$ and $j=2$, the difference coefficient and the change trend of the generalized displacement and the rate of change of the generalized displacement obtained by solving the differential coefficient differential equation was small; in $5 \mathrm{~s}-10 \mathrm{~s}$ between, the free end speed of the flexible beam at $j=1$ was larger than the value and rate of change when $j=2$; the inflection point of the speed change at $j=1$ appears earlier than when $j=2$. It can be seen from Fig. 7 that the displacement output from the free end of the flexible beam would not occur until $t=3.5 \mathrm{~s}$. When the time was $15 \mathrm{~s}$, the displacement of the flexible beam reached a stable value.

When the rotational angular velocity was $15 \mathrm{rad} / \mathrm{s}$, the generalized displacement and velocity of the beam at $j=1$ with time was obtained as shown in Fig. 8.

The curve of the generalized displacement and velocity of the free end of the beam with time at $j=2$ is shown in Fig. 9.

The curve of the vibration displacement of the free end of the beam with time is shown in Fig. 10.

When the rotational angular velocity of the rotating wheel was $15 \mathrm{rad} / \mathrm{s}$, as can be seen from Figs. 8 and 9, when $j=1$ and $j=2$, the solution for the variable coefficient differential equation was to obtain the generalized displacement and the rate of change of the generalized displacement. The difference between the value and the change trend was obvious. When $j=1$, the absolute value of the generalized displacement obtained by the solution was greater than the result obtained when $j=2$; and when $j=1$, the generalized displacement change rate at the free end of the flexible beam was greater than $j=2$; between $5 \mathrm{~s}$ and $10 \mathrm{~s}$, the free end speed of the flexible beam at $j=1$ was smaller than the value and rate of change when $j=2$; the inflection point where the speed changed greatly 


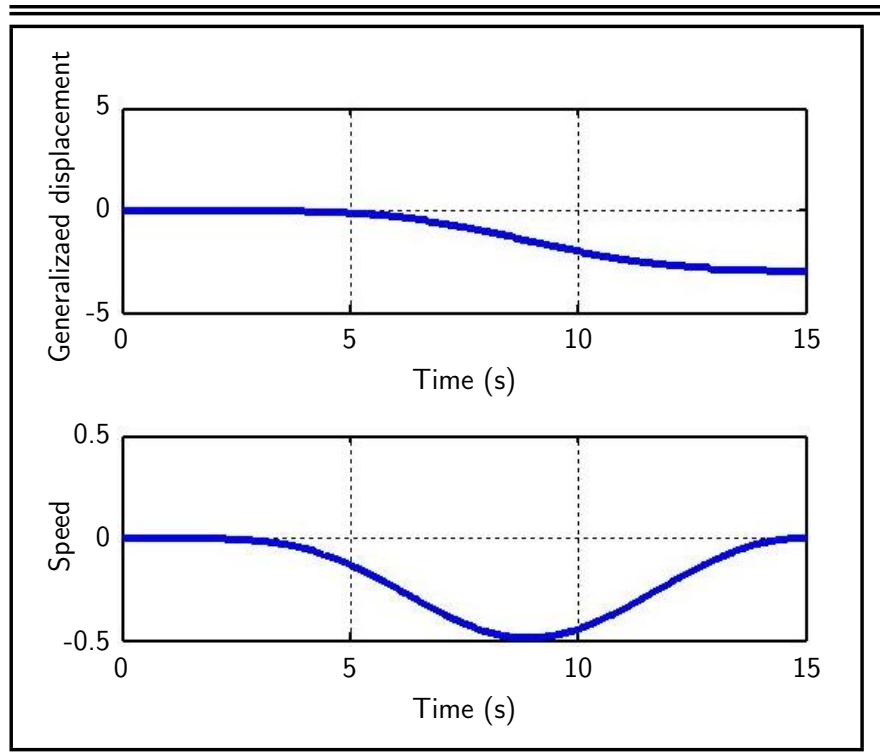

Figure 9. When $j=2$, the generalized displacement and velocity of the free end of the flexible beam.

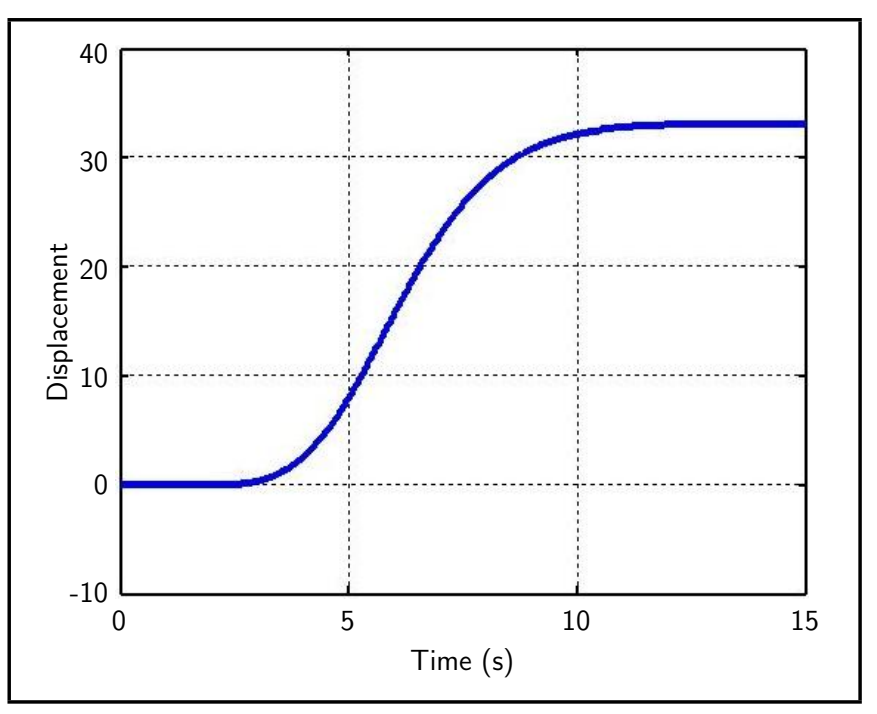

Figure 10. Vibration displacement of free end of flexible beam.

when $j=1 \mathrm{~s}$ appeared earlier than when $j=2$. It can be seen from Fig. 10 that the displacement output from the free end of the flexible beam only occurred when $t=3.5 \mathrm{~s}$. After $10 \mathrm{~s}$, the displacement of the flexible beam reached a stable value.

When the rotating wheels have different angular velocities, as the angular velocity increased, the absolute value of the displacement at the free end of the flexible beam also increased; when the rotational angular velocity reached a stable value, the displacement at the free end also reached a stable value.

\section{THE OUTPUT VOLTAGE OF THE FREE END OF THE PIEZOELECTRIC FLEXIBLE BEAM}

In the previous section, the output displacement of the free end of the flexible beam added with piezoelectric material during rotation had been obtained. According to the derived voltage output formula (29), the output value of the voltage will be

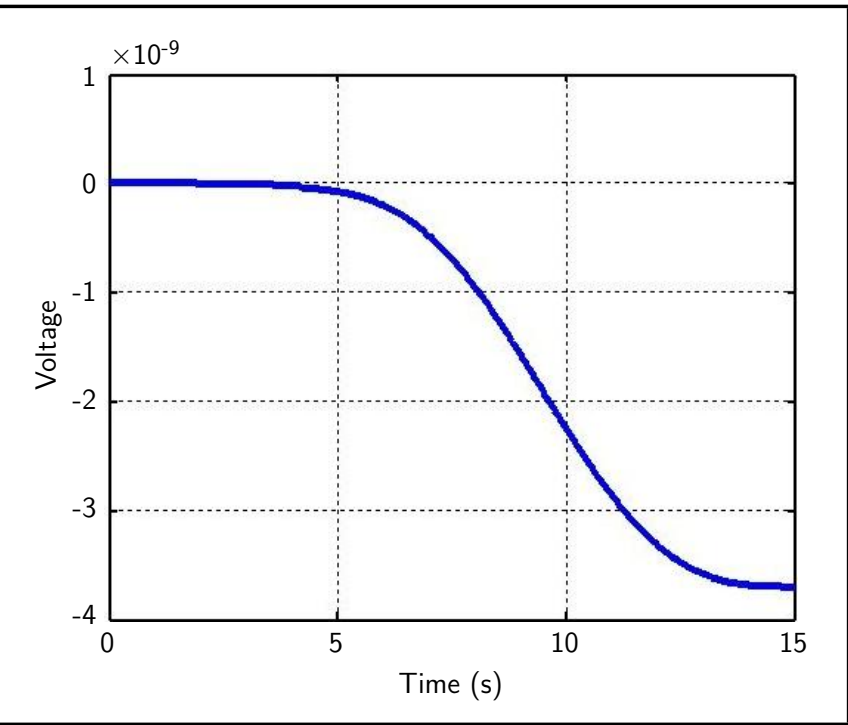

Figure 11. When the angular velocity is $10 \mathrm{rad} / \mathrm{s}$, the voltage output by the flexible beam.

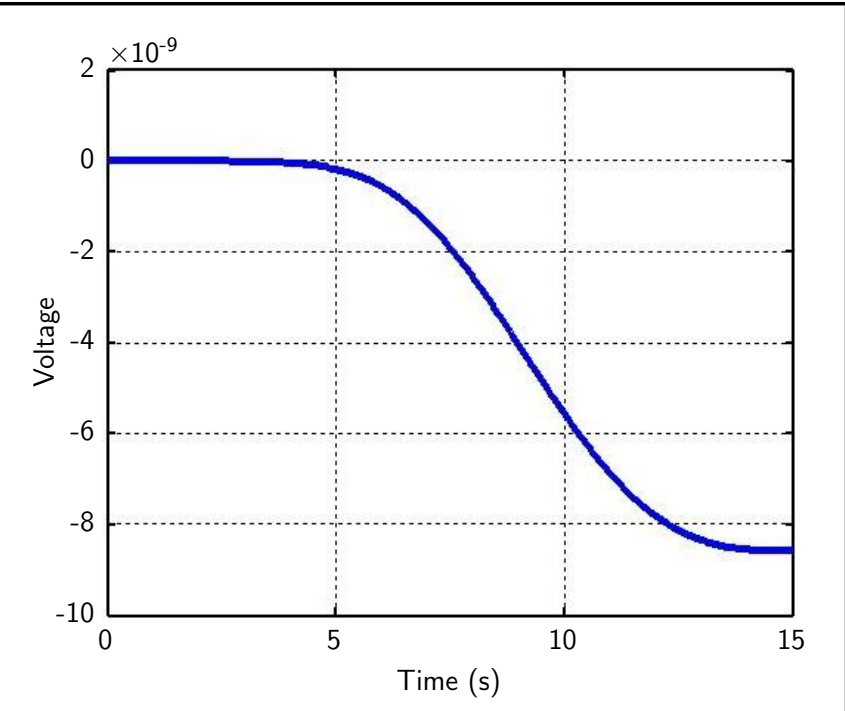

Figure 12. When the angular velocity is $15 \mathrm{rad} / \mathrm{s}$, the voltage output by the flexible beam.

determined.

In the calculation process, let $x_{2}=0.5$ and $x_{1}=0.4$. Therefore, the output voltage was the amount of change in the modal function. We still chose two different angular velocities of $10 \mathrm{rad} / \mathrm{s}$ and $15 \mathrm{rad} / \mathrm{s}$ for the rotating wheel. The output voltage of the flexible beam with piezoelectric material added at different speeds was simulated and calculated.

When the angular velocity was $10 \mathrm{rad} / \mathrm{s}$, the voltage value output by the rotating flexible beam is shown in Fig. 11.

When the angular velocity was $15 \mathrm{rad} / \mathrm{s}$, the voltage value output by the rotating flexible beam is shown in Fig. 12.

It can be seen from the curve of the output voltage value of the free end of the piezoelectric flexible beam obtained from different rotational angular velocities with time, that is, Figs. 11 and 12. When the angular velocity increased, due to the positive piezoelectricity of the piezoelectric material, the voltage generated by the effect also increased. When the angu- 
lar velocities were $10 \mathrm{rad} / \mathrm{s}$ and $15 \mathrm{rad} / \mathrm{s}$, respectively, the output voltage was more than doubled. When the rotating wheel reached a stable rotation, the voltage output by the piezoelectric material maintained a stable value.

\section{CONCLUSIONS}

(1) The fourth order Runge-Kutta method can be used to solve the differential equation of the elastic vibration variable coefficient of the flexible beam.

(2) During the rotation of the flexible beam, due to external loads such as air, air and its own "dynamic stiffening", the free end will be displaced. As the rotational angular velocity of the rotating wheel becomes larger, the displacement at the free end of the flexible beam also becomes larger. When the rotational angular velocity reaches a stable value, the displacement at the free end will also reach a stable value.

(3) Paste the piezoelectric material on the upper and lower surfaces of the cantilever flexible beam at the specified position. During the rotation process, a small voltage will be generated due to the piezoelectric effect of the piezoelectric material. Within a certain timeframe, the generated voltage becomes larger as the rotating angular velocity of the rotating wheel increases. When $t$ is $15 \mathrm{~s}$, the rotating angular velocity and voltage value tend to stabilize.

\section{ACKNOWLEDGEMENT}

The work was supported by the Shanghai Natural Science Foundation under Grant No. 19ZR1420300, and Capacity Building Projects in Local Universities of Science and Technology Commission of Shanghai Municipality (NO. 19020500900)

\section{REFERENCES}

1 Mohammadimehr, M., Rostami, R. Bending and vibration analyses of a rotating sandwich cylindrical shell considering nanocomposite core and piezoelectric layers subjected to thermal and magnetic fields [J]. Applied Mathematics and Mechanics (English Edition), 039(002): 219-240, (2018). https://dx.doi.org/10.1007/s10483-018-2301-6

2 Arefi, M., Karroubi, R., Irani-Rahaghi, M. Free vibration analysis of functionally graded laminated sandwich cylindrical shells integrated with piezoelectric layer [J]. Applied Mathematics and Mechanics (English Edition), 037(007): 821-834, (2016). https://dx.doi.org/10.1007/s10483-0162098-9
3 Cai, G. P., Lim, C. W. Dynamics studies of a flexible hub-beam system with significant damping effect $[\mathrm{J}]$. Journal of Sound and Vibration, 318(1-2): 1-17, (2008). https://dx.doi.org/10.1016/j.jsv.2008.06.009

4 Erturk, A, Inman, D J. An experimentally validated bimorph cantilever model for piezoelectric energy harvesting from base excitations [J]. Smart Materials \& Structures, 18(2): 25009-0, (2009).

5 Lu, F., Lee, H. P., Lim, S. P. Modeling and analysis of micro piezoelectric power generators for microelectromechanical-systems applications [J]. Smart Materials and Structures, 13(1): 57-63, (2004).

6 Ajitsaria, J., Choe, S. Y., Shen, D., et al. Modeling and analysis of a bimorph piezoelectric cantilever beam for voltage generation [J]. Smart Materials \& Structures, 16(2): 447, (2007).

7 Erturk, A., Inman, D. J., Issues in mathematical modeling of piezoelectric energy harvesters [J]. Smart Materials \& Structures, 17(6): 65016-0, (2008).

8 Zhang Jinfu. A new method to account for the dynamic stiffening / softening effect of flexible beams [J]. Mechanical Science and Technology, 36(8): 1161-1166, (2017), (in Chinese).

9 Zeng, S., Wang, K., Wang, B., et al. Vibration analysis of piezoelectric sandwich nanobeam with flexoelectricity based on nonlocal strain gradient theory [J]. Applied Mathematics and Mechanics, 1-22, (2020). https://dx.doi.org/10.1007/s10483-020-2620-8

10 Kerboua, M., Megnounif, A., Benguediab, M., et al. Vibration control beam using piezoelectric-based smart materials [J]. Composite Structures, 123(may): 430-442, (2015). https://dx.doi.org/10.1016/j.compstruct.2014.12.044

11 Cho, D. S., Kim, B. H., Kim, J. H., et al. Forced vibration analysis of arbitrarily constrained rectangular plates and stiffened panels using the assumed mode method [J]. Thin-Walled Structures, 90: 182-190, (2015). https://dx.doi.org/10.1016/j.tws.2015.01.020 